\title{
On formal asymptotic expansion of resonance for quantum waveguide with perforated semitransparent barrier
}

\author{
A. M. Vorobiev, A. S. Bagmutov, A. I. Popov \\ ITMO University, Kronverkskiy, 49, Staint Petersburg, 197101, Russia \\ lenden31@yandex.ru, bagmutov94@mail.ru, popov239@gmail.com
}

DOI 10.17586/2220-8054-2019-10-4-415-419

\begin{abstract}
A quantum waveguide with a semitransparent barrier, placed across it, is considered. It is assumed that the barrier has a small window. This local perturbation of the waveguide causes the appearance of resonance states localized near the barrier with the window. The asymptotics (in small parameter - the window width) of the resonances (quasi-bound states) is obtained. The procedure of construction of full formal asymptotic expansion is described. The first two terms of the asymptotic expansion are obtained explicitly. These terms describe the shift of the resonance from the threshold and the life time of the corresponding resonance state.
\end{abstract}

Keywords: quantum waveguide, resonance, asymptotics, scattering.

Received: 15 January 2019

Revised: 17 June 2019

\section{Introduction}

Problems concerning the small coupling window became interesting for mathematicians and theoretical physicists after Rayleigh's work in 1916 [1]. He considered the Helmholtz resonator and calculated the real part of the smallest quasi-eigenfrequency. Actually, it was not very significant result because calculating the imaginary part of quasieigenfrequencies and functions is a key point in such problems. For the first time, the imaginary part was calculated by Morse and Feshbach [2] using the Rayleigh method in 1960. They found a simple algebraic equation, which made it possible to approximately describe both components of this frequency - real and imaginary.

Related problems were widely studied in the second half of the 20-th century in connection with the development of nanotechnology and are quite popular currently. Now, these problems concern asymptotic analysis, resonances and resonance states. Such problems are characterized by a large number of different factors. The first factor is the system itself, which can contain different units like Helmholtz resonator, quantum waveguides, angles and so on. Of course we can consider systems with combination of such units; this may be system of two connected resonators [3], single resonator opened to space [4], pair of waveguides with common boundary [5] or even system of waveguides with resonators [6].

There are several other geometric factors characterizing such systems: shape of the resonator (it can be ideal square, circle or just some domain) [7], amount of dimensions (we can consider both two-dimensional and threedimensional problems or even something else) [8], the window position (this can be significant, as a window on the side of square resonator and window right on its angle is quite different) [9] and the number of windows [10]. There are also works related to eigenstates and resonances induced by coupling window between waveguides [11-13].

One more fundamental factor is type of boundary conditions. Actually, we consider the Helmholtz equation in most parts of problems, so we need to set up boundary conditions. Standard options are Dirichlet and Neumann conditions, but there are cases when more complicated conditions are considered. Borisov and Gadylshin studied changing conditions in the process of the problem [14] (see also another approach in [15]). This point is also important for the subject of this work, because we will consider semitransparent boundary with specific condition on it, depending on transparency parameter. Thus, this wide class of similar problems is very popular for mathematicians all over the world [16-19]. The last introductory word brings us to the subject of this work - the transparency of the barriers is one not so popular, but more important factor . A system of two waveguides with common semitransparent wall was studied by Exner and Kreicirik in [20]. 


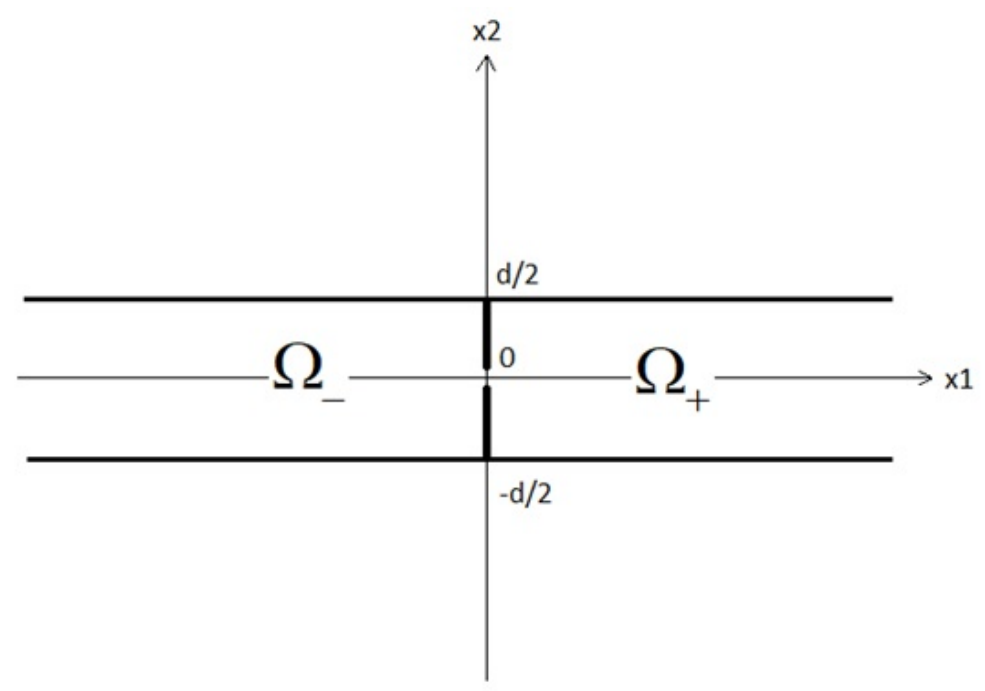

FIG. 1. The geometry of the system

\section{Asymptotics construction}

We will consider similar system, but with semitransparent barrier placed across the waveguide.

Let us set introductory formulas. $\alpha$-transparency parameter. $\alpha=0$ means no barrier, $\alpha=\infty$ - nontransparent barrier. Boundary conditions on the both waveguide walls are those of Dirichlet. But for barrier, there are specific conditions:

$$
\left\{\begin{array}{l}
u_{+}=u_{-}, \\
u_{+}^{\prime}-u_{-}^{\prime}=\alpha u .
\end{array}\right.
$$

The second condition characterizes the "jump" of the derivative on the barrier, $\alpha$ is a real constant. The conditions of such type appear if one considers singular potential supported on hypersurface. These potentials have been intensively investigated during last two decades (see, e.g., [21-25]).

The window size is $2 \varepsilon$, which is placed at the center of the barrier. For the corresponding unperturbed system (i.e., without coupling window), one can perform a separation of variables. Eigenvalues and orthonormal eigenfunctions for Laplacian (i.e. the second derivative) in the waveguide cross-section are as follows:

$$
\psi_{n}\left(x_{2}\right)=\sqrt{\frac{2}{d}} \sin \frac{\pi n x_{2}}{d}, \quad \lambda_{n}=\left(\frac{\pi n}{d}\right)^{2} .
$$

These eigenvalues play the role of the thresholds for the corresponding branches of the continuous spectrum for the waveguide Hamiltonian. The lower bound of the continuous spectrum of the Dirichlet Laplacian is greater than zero. We seek the main terms of the asymptotic expansion of a quasieigenvalues close to the first threshold:

$$
\sqrt{\left(\frac{\pi}{d}\right)^{2}-\tau_{\varepsilon}^{2}}=\sum_{j=2}^{\infty} \sum_{i=0}^{[j / 2]-1} \tau_{j i} \varepsilon^{j}\left(\ln \frac{\varepsilon}{\varepsilon_{0}}\right)^{i} .
$$

It's not the only possible expansion, but it's convenient for this case. Asymptotic series for the corresponding eigenfunctions are the following:

$$
\begin{gathered}
\psi_{\varepsilon}(x)=\left.\sqrt{\left(\frac{\pi}{d}\right)^{2}-\tau_{\varepsilon}^{2}} \cdot \sum_{j=0}^{\infty} \varepsilon^{j} P_{j+1}\left(D_{y}, \ln \frac{\varepsilon}{\varepsilon_{0}}\right) G^{-}(x, y, k)\right|_{y=0}, \quad x \in \Omega^{-} \backslash S_{\varepsilon_{0}\left(\varepsilon / \varepsilon_{0}\right)^{1 / 2}}, \\
\psi_{\varepsilon}(x)=\sum_{j=1}^{\infty} \sum_{i=0}^{[(j-1) / 2]} v_{j i}\left(\frac{x}{\varepsilon}\right) \varepsilon^{j} \ln ^{i} \frac{\varepsilon}{\varepsilon_{0}}, \quad x \in S_{2 \varepsilon_{0}\left(\varepsilon / \varepsilon_{0}\right)^{1 / 2}}, \\
\psi_{\varepsilon}(x)=-\left.\sqrt{\left(\frac{\pi}{d}\right)^{2}-\tau_{\varepsilon}^{2}} \cdot \sum_{j=0}^{\infty} \varepsilon^{j} P_{j+1}\left(D_{y}, \ln \frac{\varepsilon}{\varepsilon_{0}}\right) G^{+}(x, y, k)\right|_{y=0}, \quad x \in \Omega^{+} \backslash S_{\varepsilon_{0}\left(\varepsilon / \varepsilon_{0}\right)^{1 / 2}} .
\end{gathered}
$$


Here, $\varepsilon_{0}$ is natural unit of length, for example $d, S_{t}$ is the circle of radius $t$ with the center at the center of the window,

$n_{y}$ is normal to barrier in $\mathrm{y}$,

$$
\begin{gathered}
v_{j i} \in W_{2, l o c}^{1}\left(\Omega^{-} \cup \Omega^{+}\right), \\
P_{1}\left(D_{y}, \ln \frac{\varepsilon}{\varepsilon_{0}}\right)=c_{10}^{(1)} \frac{\partial}{\partial n_{y}},
\end{gathered}
$$

$$
\begin{gathered}
P_{m}\left(D_{y}, \ln \frac{\varepsilon}{\varepsilon_{0}}\right)=\sum_{q=1}^{m-1} \sum_{i=0}^{[(q-1) / 2]} c_{q i}^{(m)}\left(\ln \frac{\varepsilon}{\varepsilon_{0}}\right) D_{y}^{m-q+1}, \quad m \geq 2, \\
D_{y}^{2 j+1}=\frac{\partial^{2 j+1}}{\partial n_{y}^{2 j+1}}, \quad D_{y}^{2 j}=\frac{\partial^{2 j}}{\partial n_{y}^{2 j-1} \partial l_{y}} .
\end{gathered}
$$

The first thing we're going to calculate is Green's function for such system. Green's function for standard planar quantum waveguide is well known [26] and is written as:

$$
G(x, y, k)=\sum_{n=1}^{\infty} \frac{\psi_{n}\left(x_{2}\right) \cdot \psi_{n}\left(y_{2}\right)}{2 p_{n}} \cdot e^{-p_{n} \cdot\left|x_{1}-y_{1}\right|} .
$$

Here, $x_{1}$ and $y_{1}$ are coordinates on waveguide axis, $\psi$ are orthonormal eigenfunctions for non-perturbed case, $p_{n}=$ $\sqrt{\lambda_{n}-k^{2}}$ and for $n=1$ it is exactly the left part of the asymptotic expansion given before.

Let's consider our case, where waveguide is placed as in the Figure, $x_{0}$ is the abscissa of the barrier, and two arguments of Green's function are $x$ and $y$. One can write down Green's function with some coefficients considering three subdomains:

where

$$
G(x, y, k)=\sum_{n=1}^{\infty} \frac{\psi_{n}\left(x_{2}\right) \cdot \psi_{n}\left(y_{2}\right)}{2 p_{n}} \cdot \phi\left(x_{1}, y_{1}, k\right)
$$

$$
\phi\left(x_{1}, y_{1}, k\right)= \begin{cases}a_{n} \cdot e^{-p_{n} \cdot\left(x_{1}-y_{1}\right)}, & y_{1}<x_{1}, \\ b_{n} \cdot e^{-p_{n} \cdot\left(x_{1}-y_{1}\right)}+c_{n} \cdot e^{p_{n} \cdot\left(x_{1}-y_{1}\right)}, & x_{0}<x_{1}<y_{1}, \\ d_{n} \cdot e^{p_{n} \cdot\left(x_{1}-y_{1}\right)}, & x_{1}<x_{0} .\end{cases}
$$

Coefficients are calculated using conditions (1):

$$
\begin{aligned}
& \left\{\begin{array}{l}
\left.a_{n} \cdot e^{-p_{n} \cdot\left(x_{1}-y_{1}\right)}\right|_{x_{1}=y_{1}}=\left.b_{n} \cdot e^{-p_{n} \cdot\left(x_{1}-y_{1}\right)}\right|_{x_{1}=y_{1}}+\left.c_{n} \cdot e^{p_{n} \cdot\left(x_{1}-y_{1}\right)}\right|_{x_{1}=y_{1}}, \\
-\left.p_{n} a_{n} \cdot e^{-p_{n} \cdot\left(x_{1}-y_{1}\right)}\right|_{x_{1}=y_{1}}+\left.p_{n} b_{n} \cdot e^{-p_{n} \cdot\left(x_{1}-y_{1}\right)}\right|_{x_{1}=y_{1}}-\left.p_{n} c_{n} \cdot e^{p_{n} \cdot\left(x_{1}-y_{1}\right)}\right|_{x_{1}=y_{1}}=1 \\
\left.b_{n} \cdot e^{-p_{n} \cdot\left(x_{1}-y_{1}\right)}\right|_{x_{1}=y_{1}}+\left.c_{n} \cdot e^{p_{n} \cdot\left(x_{1}-y_{1}\right)}\right|_{x_{1}=y_{1}}=\left.d_{n} \cdot e^{p_{n} \cdot\left(x_{1}-y_{1}\right)}\right|_{x_{1}=y_{1}}, \\
-\left.p_{n} b_{n} \cdot e^{-p_{n} \cdot\left(x_{1}-y_{1}\right)}\right|_{x_{1}=y_{1}}+\left.p_{n} c_{n} \cdot e^{p_{n} \cdot\left(x_{1}-y_{1}\right)}\right|_{x_{1}=y_{1}}-\left.p_{n} d_{n} \cdot e^{p_{n} \cdot\left(x_{1}-y_{1}\right)}\right|_{x_{1}=y_{1}}=\left.\alpha \cdot d_{n} \cdot e^{p_{n} \cdot\left(x_{1}-y_{1}\right)}\right|_{x_{1}=y_{1}}
\end{array}\right. \\
& \left\{\begin{array} { l } 
{ a _ { n } = b _ { n } + c _ { n } , } \\
{ p _ { n } ( b _ { n } - a _ { n } - c _ { n } ) = 1 , } \\
{ b _ { n } \frac { 1 } { \gamma } + c _ { n } \gamma = d _ { n } \gamma , } \\
{ 2 c _ { n } p _ { n } = d _ { n } ( \alpha + 2 p _ { n } ) }
\end{array} \Leftrightarrow \left\{\begin{array}{l}
a_{n}=\frac{\alpha \gamma^{2}-\alpha-2 p_{n}}{2 p_{n}\left(\alpha+2 p_{n}\right)} \\
b_{n}=\frac{\alpha \gamma^{2}}{2 p_{n}\left(\alpha+2 p_{n}\right)} \\
c_{n}=-\frac{1}{2 p_{n}} \\
d_{n}=-\frac{1}{\alpha+2 p_{n}}
\end{array}\right.\right.
\end{aligned}
$$

where $\gamma=e^{p_{n} \cdot\left(x_{0}-y_{1}\right)}$. Let's notice that for no-barrier case $(\alpha=0)$ we will obtain coefficients which satisfy conventional formulas:

$$
a_{n}=c_{n}=d_{n}=-\frac{1}{2 p_{n}}, \quad b_{n}=0 .
$$

The derivative of Green's function is used in (3) and (5), thus we need the following representation:

$$
D_{y}^{j} G^{ \pm}(x, 0, k)=\left.\frac{1}{d} \sin \frac{\pi x_{2}}{d} \sin \frac{\pi y_{2}}{d} D_{y}^{j}\left(\phi\left(x_{1}, y_{1}, k\right)\right)\right|_{y_{1}=y_{0}}\left(\left(\frac{\pi}{d}\right)^{2}-k^{2}\right)^{-1 / 2}+\Phi_{j}(x, k) \ln \frac{r}{\varepsilon_{0}}+g_{j}^{ \pm}(x, k),
$$

where $g_{j}^{ \pm}(x, k)$ has no singularity at $x=0$. 
Boundary problems for coefficients $v_{j i}$ can be obtained by the following manner. Let's consider asymptotic series of $\tau_{\varepsilon}^{2}$ based on (2):

$$
\tau_{\varepsilon}^{2}=\sum_{p} \sum_{q} \Lambda_{p q} \varepsilon^{p} \ln ^{q} \frac{\varepsilon}{\varepsilon_{0}}
$$

where coefficients $\Lambda_{p q}$ are polynomials of $\tau_{j i}$ which can be easily calculated. Then, one can substitute (4) and (6) into the Helmholtz equation, change variables $\xi=\frac{x}{\varepsilon}$ and match terms of corresponding orders in the both series. Hence, one obtains the following equation:

$$
\begin{aligned}
& \Delta_{\xi} v_{j i}=-\sum_{p=0}^{j-3} \sum_{q=0}^{[p / 2]-1} \Lambda_{p q} v_{j-p-2, i-q}, \quad \xi \in R^{2} \backslash \Gamma_{1}, \\
& v_{j i}=0, \quad \xi \in \Gamma_{1},
\end{aligned}
$$

where

$$
\Gamma_{1}=\left\{\xi \mid \xi_{1}=0 \wedge \xi_{2} \in(-\infty ;-1] \cup[1 ;+\infty)\right\}
$$

Let us define operator $\mathrm{K}_{p q}$ for sums $S(x, \varepsilon)$ like (3) and (5) by the following method: if $S(x, \varepsilon)$ has coefficient $\mu(\xi)$ for $\varepsilon^{p} \ln ^{q} \frac{\varepsilon}{\varepsilon_{0}}$ in asymptotic expansion, then $\mathrm{K}_{p q}(S)=\mu$. Also let's define $\mathrm{K}_{p}=\sum_{q} \mathrm{~K}_{p q}$.

Taking into account Green's function derivative representation, we can use a procedure analogous to that in [27] and [28] and obtain:

$$
\begin{gathered}
\lim _{k \rightarrow \frac{\pi}{d}}\left(\sqrt{\left(\frac{\pi}{d}\right)^{2}-k^{2}} P_{1} G^{-}(x, 0, k)\right)=-\frac{\pi}{d^{2}} c_{10}^{(1)} \sin \frac{\pi x_{2}}{d}, \\
\lim _{k \rightarrow \frac{\pi}{d}}\left(\sqrt{\left.\left(\frac{\pi}{d}\right)^{2}-k^{2} P_{1} G^{+}(x, 0, k)\right)=0}\right. \\
\varepsilon^{-1} \mathrm{~K}_{1}\left(\sqrt{\left(\frac{\pi}{d}\right)^{2}-\tau_{\varepsilon}^{2}} \cdot P_{1} G^{+}\left(x, 0, \tau_{\varepsilon}\right)\right)= \\
=\varepsilon^{-1} c_{10}^{(1)} \mathrm{K}_{1}\left(\left(\tau_{20} \varepsilon^{2}+\tau_{30} \varepsilon^{3}+\tau_{40} \varepsilon^{4}+\tau_{41} \varepsilon^{4} \ln \frac{\varepsilon}{\varepsilon_{0}}+\ldots\right) \cdot \frac{\partial}{\partial x_{1}}\left(\frac{1}{\pi} \ln \rho+g\left(x, 0, \tau_{\varepsilon}\right)\right)\right)= \\
=\varepsilon^{-1} c_{10}^{(1)} \cdot \tau_{20} \cdot \frac{x_{1}}{\pi \rho^{2}}=\xi_{1} \cdot c_{10}^{(1)} \cdot \tau_{20} \cdot \pi^{-1} \rho^{-2} .
\end{gathered}
$$

Lemma. There exist harmonic functions $Y_{q}(\xi)$ which have the following differentiable asymptotics in $\rho \rightarrow \infty$ :

$$
Y_{q}=\left\{\begin{array}{l}
-\sum_{j=1}^{\infty} \rho^{-j}\left(a_{q j}^{+} \cos j \theta+b_{q j}^{+} \sin j \theta\right), \quad \xi_{1}>0 \\
\rho^{q}\left(a_{q}^{0} \cos q \theta+b_{q}^{0} \sin q \theta\right)+\sum_{j=1}^{\infty} \rho^{-j}\left(a_{q j}^{-} \cos j \theta+b_{q j}^{-} \sin j \theta\right), \quad \xi_{1}<0 .
\end{array}\right.
$$

To match terms from (8), we can choose $v_{10}$ as follows:

$$
v_{10}(\xi)=c_{10}^{(1)} \sum_{j=1}^{\infty} \rho^{-j}\left(a_{q j}^{+} \cos j \theta+b_{q j}^{+} \sin j \theta\right) .
$$

Now, we can equate coefficients of $\rho^{-1} \cos \theta$ in (8) and (9) to obtain $\tau_{20}$ :

$$
\begin{gathered}
\xi_{1} \cdot c_{10}^{(1)} \cdot \tau_{20} \cdot \pi^{-1} \rho^{-2}=\rho \cos \theta \cdot c_{10}^{(1)} \cdot \tau_{20} \cdot \pi^{-1} \rho^{-2}=\rho^{-1} \cos \theta \cdot c_{10}^{(1)} \cdot \tau_{20} \cdot \pi^{-1} \Rightarrow \\
\tau_{20} \cdot \pi^{-1}=a_{q 1}^{+} \Rightarrow \tau_{20}=\pi \cdot a_{q 1}^{+} .
\end{gathered}
$$

\section{Conclusion}

The suggested procedure can be continued to obtain terms of the asymptotic expansion of any order. The results concerning the real part of the resonance, give one an estimation of the shift of resonance with respect to the threshold. As for the last formula for $\tau_{20}$, it shows the imaginary part of the resonance which corresponds to the decay rate for the resonance state, i.e. to the lifetime of the quasi-bound state. These results can be useful for the description of "quantum waveguide - quantum dot - quantum waveguide" systems. One can find such systems in different nanotechnology-based applications. 


\section{Acknowledgment}

This work was partially financially supported by the Government of the Russian Federation (grant 08-08), by grant 16-11-10330 of Russian Science Foundation.

\section{References}

[1] Lord Rayleigh O.M. The theory of Helmholtz Resonator. Proceeding of Royal Society, 1916, 638, P. 265-275.

[2] Morse F.M., Feshbach G. Methods of theoretical physics, V. 2. .: Foreign Literature Publishing House, 1960, 986 p.

[3] Kiselev A.A., Pavlov B.S. The eigenfrequencies and eigenfunctions of the Laplace operator of the Neumann problem in a system of two coupled resonators. Theor. Math. Phys., 1994, 100(3), P. 354-366.

[4] Gadylshin R.R. The existence and asymptotics of poles with a small imaginary part for the Helmholtz resonator. Uspekhi of Mathematical Sciences, 1997, 52(313), P. 71-72.

[5] Borisov D.I. Discrete spectrum of an asymmetric pair of waveguides coupled through a window. Sb. Math., 2006, 197(4), P. 475-504.

[6] Achilleos V., Richoux O., et.al. Acoustic solitons in waveguides with Helmholtz resonators: Transmission line approach. Phys.Rev. E., 2015, 91, P. 023204.

[7] Martinez A., Nédélec L. Optimal lower bound of the resonance widths for a Helmoltz tube-shaped resonator. J. Spectral Th., 2012, 2, P. 203223.

[8] Gadyl'shin R.R. A two-dimensional analogue of the Helmholtz resonator with ideally rigid walls. Diff. Uravn., 1994, 30(2), P. $221-229$. Translation in Diff. Eq., 1994, 30(2), P. 201-209.

[9] Gadyl'shin R.R. Influence of the position of the opening and its shape on the properties of a Helmholtz resonator. Theor. Math. Phys., 1992, 93, P. 1151-1159.

[10] Borisov D., Exner P. Distant perturbation asymptotics in window-coupled waveguides. I. The non-threshold case. J. Math. Phys., 2006, 47(11), P. 113502(1-24).

[11] Exner P., Seba P., Tater M., Vanek D. Bound states and scattering in quantum waveguides coupled laterally through a boundary window. $J$. Math. Phys., 1996, 37, P. 4867-4887.

[12] Exner P., Vugalter S.A. Asymptotic estimates for bound states in quantum waveguides coupled laterally through a narrow window. Ann. Inst. H. Poincare, 1996, 65, P. 109-123.

[13] Popov I.Y. Asymptotics of bound state for laterally coupled waveguides. Rep. Math. Phys. 1999, 43, P. 427-437.

[14] Borisov D.I., Gadyl'shin R.R. On the spectrum of the Laplacian with frequently alternating boundary conditions. Theor. Math. Phys., 1999, 118(3), P. 272-277.

[15] Botman S.A., Leble S.B. Kinetic model of electron transport in cylindrical nanowire with rough surface. Nanosystems: Phys. Chem. Math., 2018, 9(2), P. 206-211.

[16] Popov I.Yu. The operator extension theory, semitransparent surface and short range potential. Math. Proc. Cambridge Phil. Soc., 1995, 118, P. 555-563.

[17] Popov I.Yu. The extension theory and the opening in semitransparent surface. J. Math. Phys., 1992, 33(5), P. 1685-1689.

[18] Ikebe T., Shimada S. Spectral and scattering theory for the Scgrödinger operator with penetrable wall potentials. J.Math. Kyoto Univ., 1991, 31(1), P. 219-258.

[19] Popov I.Yu. The extension theory, domains with semitransparent surface and the model of quantum dot. Proc. Royal Soc. London A., 1996, 452, P. 1505-1515.

[20] Exner P., Kreicirik D. Waveguides coupled through a semitransparent barrier: a Birman-Schwinger analysis. Rev. Math. Phys., 2001, 13, P. 307-334.

[21] Behrndt J., Langer M., Lotoreichik V. Boundary triples for Schrödinger operators with singular interactions on hypersurfaces. Nanosystems: Phys. Chem. Math., 2016, 7(2), P. 290-302.

[22] Mantile A., Posilicano A. Laplacians with singular perturbations supported on hypersurfaces. Nanosystems: Phys. Chem. Math., 2016, 7(2), P. 315-323.

[23] Exner P., Kondej S., Lotoreichik V. Asymptotics of the bound state induced by delta-interaction supported on a weakly deformed plane. $J$. Math. Phys., 2018, 59, P. 013051.

[24] Behrndt J., Exner P., et.al. Approximation of Schroedinger operators with delta-interactions supported on hypersurfaces. Math. Nachr., 2017, 290, P. 12151248.

[25] Popov I.Yu. The operator extension theory, semitransparent surface and short range potential. Math. Proc. Cambridge Phil. Soc., 1995, 118, P. 555-563.

[26] Tikhonov A.N., Samarskii A.A. Equations of Mathematical Physics. M.: Science, 1972, 531 p.

[27] Frolov S.V., Popov I.Yu. Resonances for laterally coupled quantum waveguides. J. Math. Phys., 2000, 41, P. 4391-4405.

[28] Gadyl'shin R.R. Surface potentials and the method of matching asymptotic expansions in the Helmholtz resonator problem. Algebra $i$ Analiz, 1992, 4(2), P. 88-115; translation in St. Petersburg Math. J., 1993, 4(2), P. 273-296. 